\title{
Effects of Sublabeled Rates of Dazomet and Metam-Sodium Applied Under Low-Permeability Films on Calonectria Microsclerotia Survival
}

Dalia Aiello and Alessandro Vitale, ${ }^{\dagger}$ Dipartimento di Agricoltura, Alimentazione e Ambiente, Università degli Studi di Catania; Rafael F.
Alfenas, Instituto de Ciências Agrárias e Ambientais, Universidade Federal do Mato Grosso, Sinop, Mato Grosso, Brazil; Acelino C.
Alfenas, Departamento de Fitopatologia, Universidade Federal de Viçosa UFV, Viçosa, Minas Gerais, Brazil; and Gabriella Cirvilleri and Giancarlo Polizzi, Dipartimento di Agricoltura, Alimentazione e Ambiente, University of Catania, 95123 Catania, Italy

\begin{abstract}
Infested soil is the primary inoculum source for Calonectria spp. for initiating disease in ornamental and forestry crops. The effects of dazomet and metam-sodium on survival of microsclerotia of 28 isolates belonging to 19 Calonectria spp. were evaluated in this study under nursery conditions. Two experiments with exotic Calonectria spp. in plastic containers in a greenhouse and three trials with endemic species in field plots were performed during different seasons. The containers and plots were artificially infested with Calonectria microsclerotia differentiated on carnation leaf tissues. Basamid (dazomet) was applied at 100, 160, 200, 400, and $500 \mathrm{~kg} / \mathrm{ha}$, while Divapan (metam-sodium) was applied at 250, 350, 400, 700, and 1,000 liters/ha in both the containers and plots. The fumigants were applied under virtually and totally impermeable films. Fungal survival was evaluated after 21 days using leaf tissues collected from treated soil and plated on potato dextrose agar, and the ability of microsclerotia to cause infection was tested on red clover. The survival of Calonectria inocula and microsclerotia decreased with increasing fumigant rates. In the

greenhouse trials, where Basamid was applied at 200, 400, and $500 \mathrm{~kg} / \mathrm{ha}$ and Divapan at 400, 700, and 1,000 liters/ha, no viable microsclerotia were recovered for 14 exotic Calonectria spp., whereas viable inocula of Calonectria hongkongensis, $C$. naviculata, and $C$. sulawesiensis were retrieved from the fumigated plots. Low rates of Basamid (100 and $160 \mathrm{~kg} / \mathrm{ha}$ ) and Divapan (250 and 350 liters/ha) were less effective at reducing Calonectria viability and, for these treatments, the rate of microsclerotia survival was highly variable among the different isolates and species. Furthermore, totally impermeable film significantly enhanced fumigant performance. Relative to endemic Calonectria spp., all of the treatments killed microsclerotia of $C$. polizzii and $C$. pauciramosa independent from fumigant, rate, and film. This research demonstrated the possibility of reducing the application rates by up to $160 \mathrm{~kg} / \mathrm{ha}$ for Basamid and 400 liters/ha for Divapan under low-permeability films (virtually impermeable film or totally impermeable film) for eradicating or reducing the primary inoculum of Calonectria spp. in soil.
\end{abstract}

Calonectria spp. (also known as Cylindrocladium, asexual morph) are widely distributed in tropical and subtropical climates and are pathogens of a broad range of ornamental and forestry crops, especially in nursery settings (Alfenas et al. 2013; Crous 2002; Crous et al. 1991; Lombard et al. 2009, 2010a,b, 2011; Polizzi and Crous 1999; Vitale et al. 2013b). Disease symptoms associated with Calonectria spp. include damping-off, blight, leaf spots, crown, and collar and root rot (Crous 2002; Henricot and Culham 2002; Lombard et al. 2010a; Polizzi et al. 2006a,b, 2007a,b, 2009, 2012; Vitale and Polizzi 2008; Vitale et al. 2008, 2009a). The management of infections in nurseries should involve the development of integrated strategies aimed at reducing both the level of the primary inoculum and the rate of infection in contaminated substrate or soil used for cultivation. Chemical control is the most widespread approach for managing Calonectria diseases in nurseries (Aiello et al. 2013; Cinquerrui et al. 2017; LaMondia 2014, 2015). However, the use of some fungicides such as benzimidazoles and sterol demethylation inhibitors should be limited because they induce a high-selective pressure for resistant isolates (Guarnaccia et al. 2014; Vitale et al. 2009b). Some biological control agents are effective against Calonectria infections but their efficacies are variable depending on the application modes and timing, as well as the target species and isolates (Daughtrey and Benson 2005; Harman 2000; Vitale et al. 2012).

${ }^{\dagger}$ Corresponding author: A. Vitale; E-mail: alevital@unict.it

Funding: This research was funded by MIUR project PON01_01611 SO. PRO.ME, "Sustainable production of potted plants in Mediterranean environment"; FIR project_2014, "Sustainable approaches for soil and substrate disinfestation against soilborne pathogens"; and Research Project 2016-2018, "Emergent Pests and Pathogens and Relative Sustainable Strategies5A722192112", financed by the University of Catania.

Accepted for publication 13 November 2017.

@ 2018 The American Phytopathological Society
A primary inoculum of Calonectria spp. consists of microsclerotia which can survive in soil for 15 years or more (Phipps and Beute 1979; Thies and Patton 1970). In nurseries, especially those with replants, the use of infected substrate or soil is the typical source primary Calonectria inoculum. Making matters worse, nurseries have recently begun using containers made from recycled and composted organic substrates such as peat, bark, wood fibers, and green waste (Chong 2005; Walters 2009), which can further increase the risk of Calonectria spp. infections (Noble and Roberts 2004). Once substrates are infested, the eradication of these pathogens can be very difficult. Soil solarization treatments to reduce the potential inoculum have been successfully reported against Calonectria spp. (Vitale et al. 2013a). However, the efficacy of soil solarization is strictly dependent on exposure time, the temperature reached in the upper 0 - to $30-\mathrm{cm}$ layer of soil, and the specific heat sensitivity of the targeted isolate (Vitale et al. 2013a). Soil fumigation is a more widespread practice for reducing inoculum in intensive cultivation systems; however, only limited studies have addressed Calonectria spp. infesting peanut and forest tree nurseries (Crous 2002). According to recent European directives only metam-sodium (MS), metam-potassium, and dazomet (DZ) are authorized for the disinfection of soils/ substrates in agriculture. Moreover, Directive 2009/128/EC ("Sustainable Use of Pesticide") of the European Parliament and the Council of the European Union (2009) imposes severe restrictions regarding application methods and rates, including that the fumigants should be applied at reduced rates and under mulching films that decrease gas emissions and environmental damage (European Commission 2012). As a result, our current research has focused on the more advanced multilayer films such as virtually impermeable film (VIF) and totally impermeable film (TIF) that are less permeable to chemicals than the low- and high-density polyethylene films (Fennimore and Ajwa 2011).

A preliminary study recently demonstrated the potential use of labeled rates of DZ and MS applied under VIF for the suppression of Calonectria microsclerotia in nurseries of the Mediterranean Basin (Polizzi et al. 2014). However, no data are available on the effects 
of sublabeled fumigant rates and TIF performances against epidemic Calonectria polizzii and C. pauciramosa and exotic species from forest crops. This information could improve the environmental sustainability of these fumigants.

Thus, we investigated the effects of DZ and MS applied at labeled and sublabeled rates under TIF and VIF films on 17 exotic Calonectria spp. in greenhouse trials and the effects of the fumigants applied at sublabel rates on well-established $C$. polizzii and C. pauciramosa species in the Mediterranean Basin in open fields under nursery conditions.

\section{Materials and Methods}

Pathogen isolates. In total, 28 isolates belonging to 19 Calonectria spp. were used in this study, including two species well-established in Italy, C. polizzii ITEM 14877 and C. pauciramosa ITEM 14884, and 17 exotic species previously identified by a multigene sequence analysis but not currently present in Italy (Table 1). All of the isolates were grown on potato dextrose agar (PDA; Oxoid, Basingstoke, UK) prior to transfer to carnation leaf agar (CLA) (Fisher et al. 1982) for microsclerotia production.
Effects of DZ and MS on exotic Calonectria microsclerotia. Because the exotic Calonectria spp. are not present in Italy, experiments to test fungal survival were performed in plastic containers (40 by $60 \mathrm{~cm}$ ). Two experiments (I and II) were performed in November 2014 and May 2015, respectively, in a greenhouse to determine the effects of DZ and MS on the viability of microsclerotia of 17 Calonectria spp. (26 isolates) buried in a soil substrate. The commercial fumigants evaluated were Basamid Granulat (99\% DZ, waterdispersible granules; Kanesho Soil Treatment, Brussels, Belgium) and Divapan (51\% MS, suspension concentrate; Taminco Italia s.r.l., Milano, Italia).

Microsclerotia were produced on CLA after 2 weeks of incubation at $25 \pm 1^{\circ} \mathrm{C}$, and two carnation leaf segments $(6 \mathrm{~cm}$ long $)$ colonized by pathogen microsclerotia were removed from Petri dishes and placed in a nylon mesh bag. Three bags for each isolate at each fumigant rate were buried $(15 \mathrm{~cm}$ in depth) in plastic containers filled with a loamy-sand substrate.

Basamid (DZ) was applied at rates of 100, 160, 200, 400, and $500 \mathrm{~kg} / \mathrm{ha}$ and mixed uniformly into the soil before the bags were

Table 1. Fungal isolates collected from ornamental and forest crops used in this study

\begin{tabular}{|c|c|c|c|c|c|c|}
\hline $\begin{array}{l}\text { Isolate } \\
\text { coded }^{y}\end{array}$ & Location & Host & Other collections $(\mathrm{nr} .)^{\mathrm{z}}$ & Species & Species complex & References \\
\hline LPF130 & Suzano-BA & Eucalyptus sp. & $\ldots$ & $\begin{array}{l}\text { Calonectria } \\
\text { brachiatica }\end{array}$ & C. brassicae & Lombard et al. 2009 \\
\hline LPF195 & Suzano-BA & Native forest & $\ldots$ & C. brachiatica & C. brassicae & Lombard et al. 2009 \\
\hline LPF034 & Jari & Eucalyptus sp. & $\ldots$ & C. brassicae & C. brassicae & Lombard et al. 2009 \\
\hline LPF290 & Suzano-BA & Eucalyptus sp. & $\ldots$ & C. brassicae & C. brassicae & Lombard et al. 2009 \\
\hline LPF452 & Jari & Eucalyptus sp. & $\ldots$ & C. ecuadoriae & C. brassicae & Crous et al. 2006 \\
\hline LPF300 & Jari & Eucalyptus sp. & $\ldots$ & C. orientalis & C. brassicae & $\begin{array}{l}\text { Lombard et al. } \\
\text { 2010c }\end{array}$ \\
\hline LPF301 & Jari & Eucalyptus sp. & $\ldots$ & C. orientalis & C. brassicae & $\begin{array}{l}\text { Lombard et al. } \\
\text { 2010c }\end{array}$ \\
\hline LPF031 & Jari & Eucalyptus sp. & $\ldots$ & C. pini & C. brassicae & $\begin{array}{l}\text { Lombard et al. } \\
\text { 2010c }\end{array}$ \\
\hline LPF253 & Amcel & Eucalyptus sp. & $\ldots$ & C. pini & C. brassicae & $\begin{array}{l}\text { Lombard et al. } \\
2010 \mathrm{c}\end{array}$ \\
\hline LPF388 & Suzano-MA & Eucalyptus sp. & $\ldots$ & C. brasiliensis & C. cylindrospora & $\begin{array}{l}\text { Lombard et al. } \\
\text { 2010c }\end{array}$ \\
\hline LPF244 & Viçosa-MG & Piptadenia gonoacantha & CBS133608 & C. hodgesii & C. cylindrospora & Alfenas et al. 2013 \\
\hline LPF245 & Viçosa-MG & $\begin{array}{l}\text { Anadenanthera } \\
\text { peregrina }\end{array}$ & CBS133609 & C. hodgesii & C. cylindrospora & Alfenas et al. 2013 \\
\hline LPF389 & Suzano-MA & Eucalyptus sp. & $\ldots$ & C. sulawesiensis & C. cylindrospora & $\begin{array}{l}\text { Lombard et al. } \\
\text { 2010c }\end{array}$ \\
\hline LPF007 & Jari & Eucalyptus sp. & CPC18775 & C. variabilis & C. cylindrospora & Crous et al. 1993a \\
\hline LPF220 & Amcel & Eucalyptus sp. & $\ldots$ & C. variabilis & C. cylindrospora & Crous et al. 1993a \\
\hline LPF174 & Maranhão & Eucalyptus sp. & $\ldots$ & C. ovata & C. pteridis & Victor et al. 1997 \\
\hline LPF257 & Amcel & Eucalyptus sp. & $\ldots$ & C. ovata & C. pteridis & Victor et al. 1997 \\
\hline LPF002 & Jari & Eucalyptus sp. & СРC18771 & C. pteridis & C. pteridis & Crous et al. $1993 b$ \\
\hline LPF004 & Jari & Eucalyptus sp. & CPC 18773 & C. pteridis & C. pteridis & Crous et al. 1993b \\
\hline LPF075 & Araponga-MG & Native forest & CPC18745 & C. colombiana & C. candelabra & $\begin{array}{l}\text { Lombard et al. } \\
\text { 2010c }\end{array}$ \\
\hline DISTEF173 & $\begin{array}{l}\text { Lamezia- } \\
\text { Catanzaro }\end{array}$ & Callistemon citrinus & ITEM14884 & C. pauciramosa & C. candelabra & Schoch et al. 1999 \\
\hline DISTEF87 & San Marco-ME & Acacia retinodes & ITEM14877 & C. polizzii & C. candelabra & $\begin{array}{l}\text { Lombard et al. } \\
2010 \mathrm{~b}\end{array}$ \\
\hline LPF066 & Araponga-MG & Native forest & CPC18736 & C. spathulata & C. candelabra & Crous et al. 1994 \\
\hline LPF061 & Suzano-BA & Eucalyptus sp. & $\ldots$ & C. pseudoscoparia & C. candelabra & $\begin{array}{l}\text { Lombard et al. } \\
\text { 2010c }\end{array}$ \\
\hline LPF212 & Alagoas & Eucalyptus sp. & $\ldots$ & C. pseudoscoparia & C. candelabra & $\begin{array}{l}\text { Lombard et al. } \\
\text { 2010c }\end{array}$ \\
\hline LPF219 & Suzano-BA & Native forest & $\ldots$ & C. zuluensis & C. candelabra & $\begin{array}{l}\text { Lombard et al. } \\
2010 \mathrm{~b}\end{array}$ \\
\hline LPF311 & Jari & Eucalyptus sp. & $\ldots$ & C. naviculata & $\begin{array}{l}\text { Sphaero-naviculate } \\
\text { group }\end{array}$ & Crous et al. 1994 \\
\hline LPF111 & Unknown & Unknown & $\ldots$ & C. hongkongensis & $\begin{array}{l}\text { Sphaero-naviculate } \\
\text { group }\end{array}$ & Crous et al. 2004 \\
\hline
\end{tabular}

y LPF = Laboratório de Patologia Florestal, Universidade Federal de Viçosa, Viçosa, Brazil and DISTEF = Dipartimento diAgricoltura, Alimentazione e Ambiente, Catania, Italy (Vitale et al. 2012).

${ }^{\mathrm{z}}$ CBS $=$ CBS-KNAW Fungal Biodiversity Centre, Utrecht, The Netherlands; CPC = P. W. Crous working collection housed at CBS; and ITEM = numbers as reported by Vitale et al. (2013a). 
buried, whereas Divapan (MS) was applied as soil drench with a hand sprinkler at rates of 250, 350, 400, 700, and 1,000 liters/ha. Following fumigation, the containers were brought to water field capacity, covered, and hermetically closed with VIF (Ecobrom; AgriPlast s.r.l., Vittoria, Ragusa, Italy) or TIF (Eval, Kuraray, Houston, TX) by fixing their edges at the container margins with adhesive tape. Subsequently, containers were placed in a greenhouse. The same number of bags and infected carnation leaf pieces buried in untreated substrate in plastic containers served as the controls. Three replicates (three plastic containers) were used for each isolate, rate, and tested film and arranged in a randomized complete block design applied to each fumigant separately. The nylon bags were sampled 21 days after treatment. Nine leaf pieces, obtained by cutting each leaf segment, were washed with sterile distilled water (SDW), placed onto PDA plates, and maintained for 1 week at $25 \pm 1^{\circ} \mathrm{C}$. The percentage of leaf pieces from which pathogen colonies developed was used to determine the survival of single Calonectria isolates after recovery from soil.

Effects of reduced fumigant rates on the survival of $C$. polizzii and $C$. pauciramosa microsclerotia. Experiments III, IV, and V were performed in November 2013 and May and July 2014, respectively, in open fields to determine the effects of sublabeled rates of DZ and MS on the viability of microsclerotia of $C$. polizzii and C. pauciramosa isolates. A 40-cm layer of soil was placed on a cement bed. The experimental plots, each 2.5 by $5.0 \mathrm{~m}$, were arranged in a randomized complete block design applied to each of both species separately, with three replicates for fumigant rate and tested film. Microsclerotia were produced on CLA as described above, and two carnation leaf segments $(6 \mathrm{~cm}$ long) colonized by pathogen microsclerotia were removed from Petri dishes and placed in a nylon mesh bag. Three bags for each isolate at each fumigant rate were buried (15 cm in depth) in the soil and filled with loamy-sand soil. Basamid (DZ) was applied at 100, 160, and $200 \mathrm{~kg} / \mathrm{ha}$ and mixed uniformly into the soil before the bags were buried, whereas Divapan (MS) was applied as a soil drench by a hand sprinkler at 250, 350, and 400 liters/ha. After fumigation, the soil in each plot was irrigated to field capacity and covered with sheets of VIF or TIF. The film sheets were laid on the soil surface and their edges were buried $20 \mathrm{~cm}$ deep. The same number of carnation leaf pieces buried in the untreated soil plot served as the control. At 21 days after treatment, nine leaf pieces, obtained by cutting each leaf segment, were washed with SDW, placed onto PDA plates, and maintained for 1 week at $25 \pm 1^{\circ} \mathrm{C}$. The percentage of leaf pieces from which pathogen colonies developed was used to determine the survival of Calonectria spp. after recovery from the soil.

Evaluation of the viability of $C$. polizzii and $C$. pauciramosa microsclerotia on red clover following fumigation treatments. After a 21-day exposure time to fumigation, carnation leaf samples were retrieved from both untreated and fumigated plots, and microsclerotia viability was assayed on red clover (Trifolium pratense L.) seedlings according to a method reported in recent articles (Vitale et al. 2012; Waipara et al. 1996). Additional bags containing two infected carnation leaves were previously buried in each plot (replicate) for all treatments. The retrieved carnation leaf samples were cut into smaller pieces (18 pieces per replicate) and mixed with peat substrate in an aluminum tray. The aluminum trays were placed in a growth chamber and brought to water field capacity before seeding the red clover (three replicate aluminum trays, each containing up to 70 seeds). The percentage of red clover seedlings showing crown and root rot symptoms of the total number of examined seedlings was recorded 8 to 10 days after clover seeding.

Statistical analyses. Data from independent experiments (I to V) were analyzed separately by using the Statistica package software (version 10, Analytical Software for Windows; Statsoft Inc., Tulsa, $\mathrm{OK})$. An analysis of variance was performed for each fumigant separately to evaluate the viability among Calonectria isolates exposed to single rates and to compare the performances of VIF and TIF in greenhouse experiments I and II (Table 2). For both experiments, results were analyzed in independent experiments based on year (season) and tested fumigant. Subsequently, analyses were conducted by calculating $F$ values and significances $(P)$ associated with the experimental factors (isolate, rate, and film) and whether there were significant interactions among these factors within each independent

Table 2. Analysis of variance effects of involved factors and their relative interactions on Calonectria spp. survival (\%) in plastic containers from independent experiments

\begin{tabular}{|c|c|c|c|c|c|c|}
\hline $\begin{array}{l}\text { Year } \\
\text { (season) }\end{array}$ & Fumigant & Source of variation & $\mathbf{D F}^{\mathbf{y}}$ & MS $^{\mathbf{y}}$ & $F$ value & Significance $^{\mathrm{z}}$ \\
\hline \multirow{12}{*}{$\begin{array}{l}2014 \\
\quad \text { (autumn) }\end{array}$} & Dazomet & Isolate & 25 & 1,376 & 32.8 & $* * *$ \\
\hline & $\ldots$ & Rate & 4 & 4,859 & 115.9 & $* * *$ \\
\hline & $\ldots$ & Film & 1 & 375 & 8.9 & $* *$ \\
\hline & $\ldots$ & Isolate $\times$ rate & 100 & 852 & 20.3 & $* * *$ \\
\hline & $\ldots$ & Isolate $\times$ film & 25 & 305 & 7.3 & $* * *$ \\
\hline & $\ldots$ & Isolate $\times$ rate $\times$ film & 100 & 211 & 5.0 & $* * *$ \\
\hline & Metam-sodium & Isolate & 25 & $7,294.9$ & 25.6 & $* * *$ \\
\hline & $\ldots$ & Rate & 4 & $263,479.8$ & 924.7 & $* * *$ \\
\hline & $\ldots$ & Film & 1 & $11,708.0$ & 41.1 & $* * *$ \\
\hline & $\ldots$ & Isolate $\times$ rate & 100 & $2,411.6$ & 8.5 & $* * *$ \\
\hline & $\ldots$ & Isolate $\times$ film & 25 & $1,511.7$ & 5.3 & $* * *$ \\
\hline & $\ldots$ & Isolate $\times$ rate $\times$ film & 100 & $1,178.3$ & 4.1 & $* * *$ \\
\hline \multirow{12}{*}{$\begin{array}{l}2015 \\
\text { (spring) }\end{array}$} & Dazomet & Isolate & 25 & 5,860 & 33.7 & $* * *$ \\
\hline & $\ldots$ & Rate & 4 & 52,853 & 304.4 & $* * *$ \\
\hline & $\ldots$ & Film & 1 & 7,729 & 44.5 & $* * *$ \\
\hline & $\ldots$ & Isolate $\times$ rate & 100 & 2,306 & 13.3 & $* * *$ \\
\hline & $\ldots$ & Isolate $\times$ film & 25 & 551 & 3.2 & $* * *$ \\
\hline & $\ldots$ & Isolate $\times$ rate $\times$ film & 100 & 468 & 2.7 & $* * *$ \\
\hline & Metam-sodium & Isolate & 25 & $1,920.0$ & 11.9 & $* * *$ \\
\hline & $\ldots$ & Rate & 4 & $97,912.5$ & 608.2 & $* * *$ \\
\hline & $\ldots$ & Film & 1 & $19,560.9$ & 121.5 & $* * *$ \\
\hline & $\ldots$ & Isolate $\times$ rate & 100 & $1,159.3$ & 7.2 & $* * *$ \\
\hline & $\ldots$ & Isolate $\times$ film & 25 & $1,003.0$ & 6.2 & $* * *$ \\
\hline & $\ldots$ & Isolate $\times$ rate $\times$ film & 100 & 637.2 & 3.9 & $* * *$ \\
\hline
\end{tabular}

${ }^{\mathrm{y}} \mathrm{DF}=$ degrees of freedom and MS $=$ mean square.

${ }^{\mathrm{z}}$ Asterisks $* *$ and $* * *=$ significant at $0.001<P<0.01$ and $P<0.001$, respectively. 
Table 3. Comparison of the effects of dazomet (Basamid) on microsclerotia viability reductions (\%) when applied to different Calonectria spp. in plastic containers under virtually impermeable film (VIF) or totally impermeable film (TIF) in autumn 2014 (experiment I)

\begin{tabular}{|c|c|c|c|c|c|c|}
\hline \multirow[b]{3}{*}{ Isolate } & \multicolumn{6}{|c|}{ Reductions (\%) per rate } \\
\hline & \multicolumn{2}{|c|}{$100 \mathrm{~kg} / \mathrm{ha}$} & \multicolumn{2}{|c|}{$160 \mathrm{~kg} / \mathrm{ha}$} & \multicolumn{2}{|c|}{$200 \mathrm{~kg} / \mathrm{ha}$} \\
\hline & VIF & TIF & VIF & TIF & VIF & TIF \\
\hline Calonectria brachiatica LPF130 & $100 \mathrm{a}$ & $100 \mathrm{a}$ & $100 \mathrm{a}$ & $100 \mathrm{a}$ & $100 \mathrm{a}$ & 100 \\
\hline C. brachiatica LPF195 & $100 \mathrm{a}$ & $100 \mathrm{a}$ & $100 \mathrm{a}$ & $100 \mathrm{a}$ & $100 \mathrm{a}$ & 100 \\
\hline C. brassicae LPF034 & $100 \mathrm{a}$ & $100 \mathrm{a}$ & $100 \mathrm{a}$ & $100 \mathrm{a}$ & $100 \mathrm{a}$ & 100 \\
\hline C. pini LPF253 & $100 \mathrm{a}$ & $100 \mathrm{a}$ & $100 \mathrm{a}$ & $100 \mathrm{a}$ & $100 \mathrm{a}$ & 100 \\
\hline C. orientalis LPF300 & $100 \mathrm{a}$ & $100 \mathrm{a}$ & $100 \mathrm{a}$ & $100 \mathrm{a}$ & $100 \mathrm{a}$ & 100 \\
\hline C. orientalis LPF301 & $100 \mathrm{a}$ & $100 \mathrm{a}$ & $100 \mathrm{a}$ & $100 \mathrm{a}$ & $100 \mathrm{a}$ & 100 \\
\hline C. ecuadoriae LPF452 & $100 \mathrm{a}$ & $100 \mathrm{a}$ & $100 \mathrm{a}$ & $100 \mathrm{a}$ & $100 \mathrm{a}$ & 100 \\
\hline C. pteridis LPF004 & $100 \mathrm{a}$ & $100 \mathrm{a}$ & $100 \mathrm{a}$ & $100 \mathrm{a}$ & $100 \mathrm{a}$ & 100 \\
\hline C. ovata LPF174 & $100 \mathrm{a}$ & $100 \mathrm{a}$ & $100 \mathrm{a}$ & $100 \mathrm{a}$ & $100 \mathrm{a}$ & 100 \\
\hline C. ovata LPF257 & $100 \mathrm{a}$ & $100 \mathrm{a}$ & $100 \mathrm{a}$ & $100 \mathrm{a}$ & $100 \mathrm{a}$ & 100 \\
\hline C. sulawesiensis LPF389 & $100 \mathrm{a}$ & $86.4 \mathrm{~b}$ & $100 \mathrm{a}$ & $100 \mathrm{a}$ & $100 \mathrm{a}$ & 100 \\
\hline C. pseudoscoparia LPF061 & $100 \mathrm{a}$ & $75.3 \mathrm{c}$ & $100 \mathrm{a}$ & $100 \mathrm{a}$ & $100 \mathrm{a}$ & 100 \\
\hline C. hodgesii LPF244 & $100 \mathrm{a}$ & $100 \mathrm{a}$ & $100 \mathrm{a}$ & $100 \mathrm{a}$ & $100 \mathrm{a}$ & 100 \\
\hline C. hodgesii LPF245 & $100 \mathrm{a}$ & $100 \mathrm{a}$ & $100 \mathrm{a}$ & $100 \mathrm{a}$ & $100 \mathrm{a}$ & 100 \\
\hline C. zuluensis LPF219 & $100 \mathrm{a}$ & $100 \mathrm{a}$ & $100 \mathrm{a}$ & $100 \mathrm{a}$ & $100 \mathrm{a}$ & 100 \\
\hline C. colombiana LPF075 & $100 \mathrm{a}$ & $100 \mathrm{a}$ & $100 \mathrm{a}$ & $100 \mathrm{a}$ & $100 \mathrm{a}$ & 100 \\
\hline C. spathulata LPF066 & $98.8 \mathrm{ab}$ & $100 \mathrm{a}$ & $100 \mathrm{a}$ & $100 \mathrm{a}$ & $100 \mathrm{a}$ & 100 \\
\hline C. variabilis LPF220 & $96.3 \mathrm{ab}$ & $100 \mathrm{a}$ & $87.5 \mathrm{a}$ & $100 \mathrm{a}$ & $100 \mathrm{a}$ & 100 \\
\hline C. brasiliensis LPF388 & $93.8 \mathrm{ab}$ & $100 \mathrm{a}$ & $100 \mathrm{a}$ & $100 \mathrm{a}$ & $100 \mathrm{a}$ & 100 \\
\hline C. brassicae LPF290 & $92.6 \mathrm{ab}$ & $100 \mathrm{a}$ & $100 \mathrm{a}$ & $100 \mathrm{a}$ & $100 \mathrm{a}$ & 100 \\
\hline C. pseudoscoparia LPF 212 & $92.6 \mathrm{ab}$ & $85.2 \mathrm{bc}$ & $100 \mathrm{a}$ & $100 \mathrm{a}$ & $100 \mathrm{a}$ & 100 \\
\hline C. pini $\mathrm{LPF} 031$ & $91.4 \mathrm{ab}$ & $100 \mathrm{a}$ & $100 \mathrm{a}$ & $100 \mathrm{a}$ & $100 \mathrm{a}$ & 100 \\
\hline C. variabilis $\mathrm{LPF} 007$ & $85.2 \mathrm{~b}$ & $100 \mathrm{a}$ & $100 \mathrm{a}$ & $100 \mathrm{a}$ & $100 \mathrm{a}$ & 100 \\
\hline C. pteridis $\mathrm{LPF} 002$ & $66.7 \mathrm{c}$ & $100 \mathrm{a}$ & $100 \mathrm{a}$ & $100 \mathrm{a}$ & $100 \mathrm{a}$ & 100 \\
\hline C. hongkongensis LPF111 & $33.3 \mathrm{~d}$ & $53.1 \mathrm{~d}$ & $98.8 \mathrm{a}$ & $74.1 \mathrm{~b}$ & $100 \mathrm{a}$ & 100 \\
\hline C. naviculata LPF311 & $0 \mathrm{e}$ & $56.8 \mathrm{~d}$ & $66.7 \mathrm{~b}$ & $100 \mathrm{a}$ & $66.7 \mathrm{~b}$ & 100 \\
\hline
\end{tabular}

${ }^{\mathrm{z}}$ Each value represents the mean of three replicates, each consisting of 54 infected carnation pieces. Recovery values followed by different letters within each column are significantly different according to Fisher's least significant difference test $(\alpha=0.05)$.

Table 4. Comparison of the effects of metam-sodium (Divapan) on microsclerotia viability reductions (\%) when applied to different Calonectria spp. in plastic containers under virtually impermeable film (VIF) or totally impermeable film (TIF) in autumn 2014 (experiment I)

\begin{tabular}{|c|c|c|c|c|c|c|c|c|}
\hline \multirow[b]{3}{*}{ Isolate } & \multicolumn{8}{|c|}{ Reductions (\%) per rate } \\
\hline & \multicolumn{2}{|c|}{250 liters/ha } & \multicolumn{2}{|c|}{350 liters/ha } & \multicolumn{2}{|c|}{400 liters/ha } & \multicolumn{2}{|c|}{700 liters/ha } \\
\hline & VIF & TIF & VIF & TIF & VIF & TIF & VIF & TIF \\
\hline Calonectria brassicae LPF290 & $100 \mathrm{a}$ & $75.3 \mathrm{abc}$ & $100 \mathrm{a}$ & $79 \mathrm{bc}$ & $100 \mathrm{a}$ & $100 \mathrm{a}$ & $100 \mathrm{a}$ & $100 \mathrm{a}$ \\
\hline C. hodgesii LPF244 & $76.6 \mathrm{~b}$ & $100 \mathrm{a}$ & $98.8 \mathrm{ab}$ & $100 \mathrm{a}$ & $100 \mathrm{a}$ & $100 \mathrm{a}$ & $100 \mathrm{a}$ & $100 \mathrm{a}$ \\
\hline C. hodgesii LPF245 & $58 \mathrm{bcd}$ & $51.9 \mathrm{~b}-\mathrm{f}$ & $100 \mathrm{a}$ & $81.5 \mathrm{ab}$ & $100 \mathrm{a}$ & $100 \mathrm{a}$ & $100 \mathrm{a}$ & $100 \mathrm{a}$ \\
\hline C. colombiana LPF075 & $58 \mathrm{bc}$ & $42 \mathrm{c}-\mathrm{g}$ & $69.1 \mathrm{c}-\mathrm{f}$ & $84 \mathrm{ab}$ & $100 \mathrm{a}$ & $100 \mathrm{a}$ & $100 \mathrm{a}$ & $100 \mathrm{a}$ \\
\hline C. brassicae LPF034 & 48.2 cde & $66.7 \mathrm{a}-\mathrm{e}$ & $100 \mathrm{a}$ & $92.6 \mathrm{ab}$ & $100 \mathrm{a}$ & $100 \mathrm{a}$ & $100 \mathrm{a}$ & $100 \mathrm{a}$ \\
\hline C. pteridis LPF004 & $44.4 \mathrm{c}-\mathrm{f}$ & $46.9 \mathrm{~b}-\mathrm{f}$ & $55.6 \mathrm{fg}$ & $100 \mathrm{a}$ & $100 \mathrm{a}$ & $100 \mathrm{a}$ & $100 \mathrm{a}$ & $100 \mathrm{a}$ \\
\hline C. zuluensis LPF219 & 44.4 cde & $43.2 \mathrm{~b}-\mathrm{f}$ & $77.8 \mathrm{a}-\mathrm{f}$ & $100 \mathrm{a}$ & $100 \mathrm{a}$ & $100 \mathrm{a}$ & $100 \mathrm{a}$ & $100 \mathrm{a}$ \\
\hline C. spathulata $\mathrm{LPF} 066$ & $43.2 \mathrm{c}-\mathrm{f}$ & $28.4 \mathrm{f}-\mathrm{i}$ & $33.3 \mathrm{ghi}$ & $70.4 \mathrm{bc}$ & $100 \mathrm{a}$ & $100 \mathrm{a}$ & $100 \mathrm{a}$ & $100 \mathrm{a}$ \\
\hline C. pini LPF253 & 42 cde & $35.8 \mathrm{e}-\mathrm{h}$ & $59.3 \mathrm{efg}$ & $70.4 \mathrm{bcd}$ & $100 \mathrm{a}$ & $100 \mathrm{a}$ & $100 \mathrm{a}$ & $100 \mathrm{a}$ \\
\hline C. sulawesiensis LPF389 & $35.8 \mathrm{c}-\mathrm{f}$ & $18.5 \mathrm{f}-\mathrm{i}$ & $93.8 \mathrm{abc}$ & $76.6 \mathrm{bc}$ & $100 \mathrm{a}$ & $91.4 \mathrm{~b}$ & $100 \mathrm{a}$ & $100 \mathrm{a}$ \\
\hline C. pini LPF031 & $34.6 \mathrm{~d}-\mathrm{g}$ & $34.6 \mathrm{e}-\mathrm{h}$ & 67.9 def & $100 \mathrm{a}$ & $100 \mathrm{a}$ & $100 \mathrm{a}$ & $100 \mathrm{a}$ & $100 \mathrm{a}$ \\
\hline C. orientalis LPF300 & $32.1 \mathrm{def}$ & $55.6 \mathrm{~b}-\mathrm{f}$ & $84 \mathrm{a}-\mathrm{d}$ & $84 \mathrm{ab}$ & $100 \mathrm{a}$ & $100 \mathrm{a}$ & $100 \mathrm{a}$ & $100 \mathrm{a}$ \\
\hline C. ovata LPF 257 & $27.2 \mathrm{e}-\mathrm{h}$ & $70.4 \mathrm{a}-\mathrm{d}$ & $54.3 \mathrm{efg}$ & $100 \mathrm{a}$ & $100 \mathrm{a}$ & $100 \mathrm{a}$ & $100 \mathrm{a}$ & $100 \mathrm{a}$ \\
\hline C. pseudoscoparia LPF061 & $25.9 \mathrm{e}-\mathrm{i}$ & $33.3 \mathrm{e}-\mathrm{i}$ & $80.3 \mathrm{a}-\mathrm{e}$ & $40.7 \mathrm{e}$ & $100 \mathrm{a}$ & $100 \mathrm{a}$ & $100 \mathrm{a}$ & $100 \mathrm{a}$ \\
\hline C. orientalis LPF301 & $24.7 \mathrm{e}-\mathrm{h}$ & $50.6 \mathrm{~b}-\mathrm{f}$ & $86.4 \mathrm{a}-\mathrm{d}$ & 58 cde & $100 \mathrm{a}$ & $100 \mathrm{a}$ & $100 \mathrm{a}$ & $100 \mathrm{a}$ \\
\hline C. ovata $\mathrm{LPF} 174$ & $23.5 \mathrm{f}-\mathrm{i}$ & $77.8 \mathrm{ab}$ & $75.2 \mathrm{a}-\mathrm{d}$ & $100 \mathrm{a}$ & $100 \mathrm{a}$ & $100 \mathrm{a}$ & $100 \mathrm{a}$ & $100 \mathrm{a}$ \\
\hline C. brachiatica LPF130 & $21 \mathrm{e}-\mathrm{i}$ & $81.5 \mathrm{abc}$ & $100 \mathrm{a}$ & $100 \mathrm{a}$ & $100 \mathrm{a}$ & $100 \mathrm{a}$ & $100 \mathrm{a}$ & $100 \mathrm{a}$ \\
\hline C. variabilis LPF220 & 9.9 ghi & $100 \mathrm{a}$ & $30.9 \mathrm{gh}$ & $100 \mathrm{a}$ & $100 \mathrm{a}$ & $100 \mathrm{a}$ & $100 \mathrm{a}$ & $100 \mathrm{a}$ \\
\hline C. variabilis LPF007 & $3.7 \mathrm{hi}$ & $66.7 \mathrm{a}-\mathrm{e}$ & $72.8 \mathrm{a}-\mathrm{f}$ & $70.4 \mathrm{bc}$ & $100 \mathrm{a}$ & $100 \mathrm{a}$ & $100 \mathrm{a}$ & $100 \mathrm{a}$ \\
\hline C. pseudoscoparia LPF212 & $0 \mathrm{i}$ & $56.8 \mathrm{~b}-\mathrm{f}$ & $77.8 \mathrm{a}-\mathrm{f}$ & $69.1 \mathrm{bcd}$ & $100 \mathrm{a}$ & $100 \mathrm{a}$ & $100 \mathrm{a}$ & $100 \mathrm{a}$ \\
\hline C. brasiliensis LPF388 & $0 \mathrm{i}$ & $51.9 \mathrm{~b}-\mathrm{f}$ & $69.1 \mathrm{~b}-\mathrm{f}$ & $50.6 \mathrm{de}$ & $100 \mathrm{a}$ & $100 \mathrm{a}$ & $100 \mathrm{a}$ & $100 \mathrm{a}$ \\
\hline C. ecuadoriae LPF452 & $0 \mathrm{i}$ & $42 \mathrm{c}-\mathrm{h}$ & $100 \mathrm{a}$ & $92.6 \mathrm{ab}$ & $100 \mathrm{a}$ & $100 \mathrm{a}$ & $100 \mathrm{a}$ & $100 \mathrm{a}$ \\
\hline C. brachiatica LPF195 & $0 \mathrm{i}$ & 40.7 deh & $14.8 \mathrm{hi}$ & $53.1 \mathrm{cde}$ & $100 \mathrm{a}$ & $100 \mathrm{a}$ & $100 \mathrm{a}$ & $100 \mathrm{a}$ \\
\hline C. pteridis LPF002 & $0 \mathrm{i}$ & 7.4 ghi & $64.2 \mathrm{def}$ & $33.3 \mathrm{ef}$ & $100 \mathrm{a}$ & $100 \mathrm{a}$ & $100 \mathrm{a}$ & $100 \mathrm{a}$ \\
\hline C. naviculata LPF311 & $0 \mathrm{i}$ & $7.4 \mathrm{ghi}$ & $18.5 \mathrm{hi}$ & $11.1 \mathrm{f}$ & $100 \mathrm{a}$ & $100 \mathrm{a}$ & $100 \mathrm{a}$ & $100 \mathrm{a}$ \\
\hline C. hongkongensis LPF111 & $0 \mathrm{i}$ & $0 \mathrm{i}$ & $4.9 \mathrm{i}$ & $11.1 \mathrm{f}$ & $61.7 \mathrm{~b}$ & $13.6 \mathrm{c}$ & $93.8 \mathrm{~b}$ & $96.3 \mathrm{~b}$ \\
\hline
\end{tabular}

${ }^{\mathrm{z}}$ Each value represents the mean of three replicates, each consisting of 54 infected carnation pieces. Recovery values followed by different letters within each column are significantly different according to Fisher's least significant difference test $(\alpha=0.05)$. 
Table 5. Comparison of the effects of dazomet (Basamid) on microsclerotia viability reductions (\%) when applied to different Calonectria spp. in plastic containers under virtually impermeable film (VIF) or totally impermeable film (TIF) in spring 2015 (experiment II)

\begin{tabular}{|c|c|c|c|c|c|c|}
\hline \multirow[b]{3}{*}{ Isolate } & \multicolumn{6}{|c|}{ Reductions (\%) per rate } \\
\hline & \multicolumn{2}{|c|}{$100 \mathrm{~kg} / \mathrm{ha}$} & \multicolumn{2}{|c|}{$160 \mathrm{~kg} / \mathrm{ha}$} & \multicolumn{2}{|c|}{$200 \mathrm{~kg} / \mathrm{ha}$} \\
\hline & VIF & TIF & VIF & TIF & VIF & TIF \\
\hline Calonectria brachiatica LPF130 & $100 \mathrm{a}$ & $100 \mathrm{a}$ & $100 \mathrm{a}$ & $100 \mathrm{a}$ & $100 \mathrm{a}$ & $100 \mathrm{a}$ \\
\hline C. pteridis LPF004 & $100 \mathrm{a}$ & $100 \mathrm{a}$ & $100 \mathrm{a}$ & $100 \mathrm{a}$ & $100 \mathrm{a}$ & $100 \mathrm{a}$ \\
\hline C. ovata LPF174 & $100 \mathrm{a}$ & $100 \mathrm{a}$ & $100 \mathrm{a}$ & $100 \mathrm{a}$ & $100 \mathrm{a}$ & $100 \mathrm{a}$ \\
\hline C. zuluensis LPF219 & $100 \mathrm{a}$ & $100 \mathrm{a}$ & $100 \mathrm{a}$ & $100 \mathrm{a}$ & $100 \mathrm{a}$ & $100 \mathrm{a}$ \\
\hline C. colombiana LPF075 & $100 \mathrm{a}$ & $100 \mathrm{a}$ & $100 \mathrm{a}$ & $100 \mathrm{a}$ & $100 \mathrm{a}$ & $100 \mathrm{a}$ \\
\hline C. pseudoscoparia LPF212 & $100 \mathrm{a}$ & $100 \mathrm{a}$ & $100 \mathrm{a}$ & $100 \mathrm{a}$ & $100 \mathrm{a}$ & $100 \mathrm{a}$ \\
\hline C. variabilis $\mathrm{LPF} 007$ & $95.1 \mathrm{ab}$ & $100 \mathrm{a}$ & $100 \mathrm{a}$ & $100 \mathrm{a}$ & $100 \mathrm{a}$ & $100 \mathrm{a}$ \\
\hline C. hodgesii LPF245 & $90.1 \mathrm{abc}$ & $100 \mathrm{a}$ & $100 \mathrm{a}$ & $100 \mathrm{a}$ & $100 \mathrm{a}$ & $100 \mathrm{a}$ \\
\hline C. hodgesii LPF244 & $87.7 \mathrm{abc}$ & $88.9 \mathrm{ab}$ & $100 \mathrm{a}$ & $100 \mathrm{a}$ & $100 \mathrm{a}$ & $100 \mathrm{a}$ \\
\hline C. ovata $\mathrm{LPF} 257$ & $86.4 \mathrm{abc}$ & $19.8 \mathrm{ab}$ & $100 \mathrm{a}$ & $100 \mathrm{a}$ & $100 \mathrm{a}$ & $100 \mathrm{a}$ \\
\hline C. brassicae LPF290 & $84 \mathrm{abc}$ & $100 \mathrm{a}$ & $100 \mathrm{a}$ & $100 \mathrm{a}$ & $100 \mathrm{a}$ & $100 \mathrm{a}$ \\
\hline C. ecuadoriae LPF452 & $72.8 \mathrm{bcd}$ & $100 \mathrm{a}$ & $100 \mathrm{a}$ & $100 \mathrm{a}$ & $100 \mathrm{a}$ & $100 \mathrm{a}$ \\
\hline C. brassicae LPF034 & $71.6 \mathrm{~cd}$ & $77.8 \mathrm{ab}$ & $100 \mathrm{a}$ & $100 \mathrm{a}$ & $100 \mathrm{a}$ & $100 \mathrm{a}$ \\
\hline C. spathulata LPF066 & $66.7 \mathrm{~cd}$ & $100 \mathrm{a}$ & $100 \mathrm{a}$ & $100 \mathrm{a}$ & $100 \mathrm{a}$ & $100 \mathrm{a}$ \\
\hline C. pseudoscoparia LPF061 & $66.7 \mathrm{~cd}$ & $66.7 \mathrm{bc}$ & $100 \mathrm{a}$ & $100 \mathrm{a}$ & $100 \mathrm{a}$ & $100 \mathrm{a}$ \\
\hline C. brachiatica LPF195 & $58 \mathrm{de}$ & $100 \mathrm{a}$ & $100 \mathrm{a}$ & $100 \mathrm{a}$ & $100 \mathrm{a}$ & $100 \mathrm{a}$ \\
\hline C. pini LPF031 & $49.4 \mathrm{de}$ & $61.7 \mathrm{bc}$ & $100 \mathrm{a}$ & $100 \mathrm{a}$ & $100 \mathrm{a}$ & $100 \mathrm{a}$ \\
\hline C. pini LPF253 & $48.2 \mathrm{de}$ & $49.4 \mathrm{~cd}$ & $100 \mathrm{a}$ & $100 \mathrm{a}$ & $100 \mathrm{a}$ & $100 \mathrm{a}$ \\
\hline C. brasiliensis LPF388 & $35.8 \mathrm{ef}$ & $33.3 \mathrm{de}$ & $100 \mathrm{a}$ & $100 \mathrm{a}$ & $100 \mathrm{a}$ & $100 \mathrm{a}$ \\
\hline C. pteridis LPF002 & $34.6 \mathrm{ef}$ & $100 \mathrm{a}$ & $100 \mathrm{a}$ & $100 \mathrm{a}$ & $100 \mathrm{a}$ & $100 \mathrm{a}$ \\
\hline C. sulawesiensis LPF389 & $33.3 \mathrm{ef}$ & $66.7 \mathrm{bc}$ & $100 \mathrm{a}$ & $100 \mathrm{a}$ & $100 \mathrm{a}$ & $100 \mathrm{a}$ \\
\hline C. hongkongensis LPF111 & $21 \mathrm{f}$ & $32.1 \mathrm{de}$ & $33.3 \mathrm{c}$ & $33.3 \mathrm{c}$ & $66.7 \mathrm{~b}$ & $66.7 \mathrm{~b}$ \\
\hline C. naviculata LPF311 & $16 \mathrm{f}$ & $11.1 \mathrm{e}$ & $33.3 \mathrm{c}$ & $46.9 \mathrm{~b}$ & $56.8 \mathrm{~b}$ & $100 \mathrm{a}$ \\
\hline C. orientalis LPF300 & $11.1 \mathrm{f}$ & $69.1 \mathrm{bc}$ & $100 \mathrm{a}$ & $100 \mathrm{a}$ & $100 \mathrm{a}$ & $100 \mathrm{a}$ \\
\hline C. orientalis LPF301 & $11.1 \mathrm{f}$ & $48.2 \mathrm{~cd}$ & $100 \mathrm{a}$ & $100 \mathrm{a}$ & $100 \mathrm{a}$ & $100 \mathrm{a}$ \\
\hline C. variabilis LPF220 & $7.4 \mathrm{f}$ & $100 \mathrm{a}$ & $76.5 \mathrm{~b}$ & $100 \mathrm{a}$ & $100 \mathrm{a}$ & $100 \mathrm{a}$ \\
\hline
\end{tabular}

${ }^{\mathrm{z}}$ Each value represents the mean of three replicates, each consisting of 54 infected carnation pieces. Recovery values followed by different letters within each column are significantly different according to Fisher's least significant difference test $(\alpha=0.05)$.

Table 6. Comparison of the effects of metam-sodium (Divapan) on microsclerotia viability reductions (\%) when applied to different Calonectria spp. in plastic containers under virtually impermeable film (VIF) or totally impermeable film (TIF) in spring 2015 (experiment II)

\begin{tabular}{|c|c|c|c|c|}
\hline \multirow[b]{3}{*}{ Isolate } & \multicolumn{4}{|c|}{ Reductions (\%) per rate } \\
\hline & \multicolumn{2}{|c|}{250 liters/ha } & \multicolumn{2}{|c|}{350 liters/ha } \\
\hline & VIF & TIF & VIF & TIF \\
\hline Calonectria brassicae LPF290 & $100 \mathrm{a}$ & $79.2 \mathrm{abc}$ & $100 \mathrm{a}$ & $83.3 \mathrm{c}$ \\
\hline C. pseudoscoparia LPF212 & $80.3 \mathrm{abc}$ & $100 \mathrm{a}$ & $100 \mathrm{a}$ & $100 \mathrm{a}$ \\
\hline C. sulawesiensis LPF389 & $75.3 \mathrm{a}-\mathrm{d}$ & 70.4 cde & $100 \mathrm{a}$ & $90.1 \mathrm{~b}$ \\
\hline C. hodgesii LPF244 & $72.7 \mathrm{ab}$ & $100 \mathrm{a}$ & $100 \mathrm{a}$ & $100 \mathrm{a}$ \\
\hline C. orientalis LPF300 & $70.4 \mathrm{~b}-\mathrm{e}$ & $80.3 \mathrm{bcd}$ & $100 \mathrm{a}$ & $100 \mathrm{a}$ \\
\hline C. spathulata $\mathrm{LPF} 066$ & $69.2 \mathrm{~b}-\mathrm{e}$ & $85.2 \mathrm{abc}$ & $65.4 \mathrm{~d}-\mathrm{g}$ & $100 \mathrm{a}$ \\
\hline C. ovata $\mathrm{LPF} 257$ & $66.7 \mathrm{~b}-\mathrm{f}$ & $100 \mathrm{a}$ & $100 \mathrm{a}$ & $100 \mathrm{a}$ \\
\hline C. colombiana LPF075 & $64.2 \mathrm{~b}-\mathrm{f}$ & 70.4 cde & $100 \mathrm{a}$ & $100 \mathrm{a}$ \\
\hline C. naviculata LPF311 & $63 \mathrm{~b}-\mathrm{f}$ & $33.3 \mathrm{fgh}$ & $86.4 \mathrm{bcd}$ & $58 \mathrm{~d}$ \\
\hline C. hodgesii LPF245 & $55.6 \mathrm{~b}-\mathrm{g}$ & $86.4 \mathrm{abc}$ & $100 \mathrm{a}$ & $100 \mathrm{a}$ \\
\hline C. pini LPF253 & $51.9 \mathrm{c}-\mathrm{g}$ & $63 \mathrm{c}-\mathrm{f}$ & $100 \mathrm{a}$ & $100 \mathrm{a}$ \\
\hline C. ovata LPF174 & $50.6 \mathrm{c}-\mathrm{g}$ & $85.2 \mathrm{abc}$ & $58.1 \mathrm{efg}$ & $100 \mathrm{a}$ \\
\hline C. pteridis LPF004 & $49.4 \mathrm{e}-\mathrm{h}$ & $84 \mathrm{abc}$ & $92.6 \mathrm{abc}$ & $100 \mathrm{a}$ \\
\hline C. brachiatica LPF130 & $49.4 \mathrm{c}-\mathrm{g}$ & $63 \mathrm{c}-\mathrm{f}$ & $100 \mathrm{a}$ & $100 \mathrm{a}$ \\
\hline C. brachiatica LPF195 & $45.7 \mathrm{c}-\mathrm{g}$ & $95.1 \mathrm{ab}$ & 40.7 efg & $100 \mathrm{a}$ \\
\hline C. zuluensis LPF219 & $44.5 \mathrm{e}-\mathrm{h}$ & $88.9 \mathrm{ab}$ & $100 \mathrm{a}$ & $100 \mathrm{a}$ \\
\hline C. brassicae LPF034 & $44.4 \mathrm{~d}-\mathrm{h}$ & $100 \mathrm{a}$ & $100 \mathrm{a}$ & $100 \mathrm{a}$ \\
\hline C. brasiliensis LPF388 & $42 \mathrm{e}-\mathrm{h}$ & $54.3 \mathrm{~d}-\mathrm{g}$ & $86.4 \mathrm{abc}$ & $98.8 \mathrm{ab}$ \\
\hline C. orientalis LPF301 & $42 \mathrm{e}-\mathrm{h}$ & $44.5 \mathrm{e}-\mathrm{h}$ & $87.7 \mathrm{abc}$ & $100 \mathrm{a}$ \\
\hline C. variabilis LPF220 & $35.8 \mathrm{fgh}$ & $100 \mathrm{a}$ & $74.1 \mathrm{c}-\mathrm{f}$ & $100 \mathrm{a}$ \\
\hline C. variabilis LPF007 & $34.6 \mathrm{gh}$ & $98.8 \mathrm{ab}$ & 88.9 abc & $100 \mathrm{a}$ \\
\hline C. pini LPF031 & $33.3 \mathrm{gh}$ & $81.5 \mathrm{abc}$ & $51.9 \mathrm{fg}$ & $100 \mathrm{a}$ \\
\hline C. ecuadoriae LPF452 & $32.1 \mathrm{fgh}$ & $48.1 \mathrm{efg}$ & $100 \mathrm{a}$ & $100 \mathrm{a}$ \\
\hline C. hongkongensis LPF111 & $32.1 \mathrm{gh}$ & $30.9 \mathrm{~h}$ & $51.9 \mathrm{~g}$ & $38.3 \mathrm{e}$ \\
\hline C. pseudoscoparia LPF061 & $22.2 \mathrm{hi}$ & $100 \mathrm{a}$ & $97.5 \mathrm{ab}$ & $100 \mathrm{a}$ \\
\hline C. pteridis LPF002 & $0 \mathrm{i}$ & $33.3 \mathrm{gh}$ & 79 cde & $100 \mathrm{a}$ \\
\hline
\end{tabular}

${ }^{\mathrm{z}}$ Each value represents the mean of three replicates, each consisting of 54 infected carnation pieces. Recovery values followed by different letters within each column are significantly different according to Fisher's least significant difference test $(\alpha=0.05)$. 
experiment. The efficacy of the fumigants and rates on $C$. polizzii ITEM 14877 and $C$. pauciramosa ITEM 14884 microsclerotia viability and the relative VIF and TIF performances at each fumigant rate in experiments III, IV, and V in the soil under open-field conditions were also examined using an analysis of variance. The mean separation of the viability reduction percentages compared with relative controls in plastic containers among isolates, the fumigant effects on $C$. polizzii and $C$. pauciramosa survival rates in soil under open-field conditions, and the relative infectivity levels (disease incidence) in red clover were assessed using Fisher's least significance difference test at $\alpha=0.05$.

\section{Results}

Effects of DZ and MS on exotic Calonectria microsclerotia. Isolate, rate, film, and their interactions always significantly affected the number of viable microsclerotia buried in the fumigated plots (Table 2). TIF significantly enhanced the performance of fumigation when compared with VIF over independent experiments (Table 2). Data from experiments I and II are reported in Tables 3, 4, 5, and 6).

Because all of the interactions were significant (Table 2), the responses to fumigation among the isolates were analyzed and shown for single fumigation rates under the same film (Tables 3, 4, 5, and 6).

In experiment I, Basamid (DZ) at 400 and $500 \mathrm{~kg} / \mathrm{ha}$ resulted in the elimination of microsclerotia viability for all of the Calonectria spp. whereas, at $200 \mathrm{~kg} / \mathrm{ha}$, viability was eliminated in all of the species, except $C$. naviculata. When this fumigant was applied at $160 \mathrm{~kg} / \mathrm{ha}$, no viable microsclerotia were retrieved from fumigated containers for any species, except for C. variabilis LPF220, C. hongkongensis, and $C$. naviculata isolates. This fumigant was less effective when applied at $100 \mathrm{~kg} / \mathrm{ha}$, resulting in the elimination of microsclerotia viability for 16 of 26 and 21 of 26 isolates under VIF and TIF, respectively. The highest variability of response to $\mathrm{DZ}$ fumigation was detected at $100 \mathrm{~kg} / \mathrm{ha}$ of the commercial formulate (Basamid; Table 3). Divapan (MS) at 1,000 liters/ha eliminated the microsclerotia viability of all species or isolates whereas, at 700 and 400 liters/ha, viable inocula were recovered only from $C$. sulawesiensis and $C$. hongkongensis. The commercial formulate (Divapan) at 250 and 350 liters/ha showed weaker capabilities to reduce the microsclerotia viability of Calonectria spp. or isolates, and these concentrations resulted in the greatest variability in response to fumigation among the tested isolates (Table 4).

In experiment II, the highest rates of Basamid (DZ) showed excellent efficacies at 400 and $500 \mathrm{~kg} / \mathrm{ha}$ in killing the microsclerotia of all Calonectria spp. This fumigant applied at 160 and $200 \mathrm{~kg} / \mathrm{ha}$ totally reduced the microsclerotia viability of all species, except for $C$. naviculata, C. variabilis LPF220, and C. hongkongensis, whereas, at $100 \mathrm{~kg} / \mathrm{ha}$, it was, on average, less effective compared with the data of experiment I. At this fumigant rate, the elimination of microsclerotia viability was detected for only 6 of 26 and 14 of 26 isolates under VIF and TIF, respectively (Table 5).

Divapan (MS) at 400, 700, and 1,000 liters/ha eliminated Calonectria microsclerotia viability, whereas the 350 liters/ha rate killed the most Calonectria isolates under TIF (21 of 26) and half of the isolates under VIF. The commercial formulate applied at 250 liters/ha was the least efficient in reducing the viability of Calonectria inocula. As in the previous experiment, a high rate of reduction in viability among $\mathrm{Cal}$ onectria isolates was detected at the low fumigation rates (Table 6).

Effects of reduced fumigant rates on the survival of $C$. polizzii and $C$. pauciramosa microsclerotia. Data from experiments III, IV, and $\mathrm{V}$, performed in open fields, are reported in Table 7. In experiment III, all of the treatments killed microsclerotia of $C$. polizzii

Table 7. Effectiveness of reduced rates of dazomet (Basamid) and metam-sodium (Divapan) applied to loamy-sand soil in affecting survival (\%) of Calonectria polizzii and C. pauciramosa from infected carnation leaves after exposure to fumigation treatment under virtually impermeable film (VIF) or totally impermeable film (TIF) soil mulchings in the nursery in three experiments ${ }^{y}$

\begin{tabular}{|c|c|c|c|c|c|c|c|c|c|c|c|c|}
\hline \multirow{3}{*}{$\begin{array}{l}\text { Treatment, } \\
\text { rate }^{\mathrm{z}}\end{array}$} & \multicolumn{4}{|c|}{ Experiment III (summer 2013) } & \multicolumn{4}{|c|}{ Experiment IV (autumn 2013) } & \multicolumn{4}{|c|}{ Experiment V (spring 2014) } \\
\hline & \multicolumn{2}{|c|}{ C. polizzii } & \multicolumn{2}{|c|}{ C.pauciramosa } & \multicolumn{2}{|c|}{ C. polizzii } & \multicolumn{2}{|c|}{ C. pauciramosa } & \multicolumn{2}{|c|}{ C. polizzii } & \multicolumn{2}{|c|}{ C. pauciramosa } \\
\hline & VIF & TIF & VIF & TIF & VIF & TIF & VIF & TIF & VIF & TIF & VIF & TIF \\
\hline Divapan (400) & 0 & 0 & 0 & 0 & $0 \mathrm{a}$ & $0 \mathrm{a}$ & $0 \mathrm{a}$ & $0 \mathrm{a}$ & $0 \mathrm{a}$ & $0 \mathrm{a}$ & $0 \mathrm{a}$ & $0 \mathrm{a}$ \\
\hline Divapan (350) & 0 & 0 & 0 & 0 & $0 \mathrm{a}$ & $0 \mathrm{a}$ & $0 \mathrm{a}$ & $0 \mathrm{a}$ & $0 \mathrm{a}$ & $0 \mathrm{a}$ & $0 \mathrm{a}$ & $0 \mathrm{a}$ \\
\hline Divapan (250) & 0 & 0 & 0 & 0 & $9.9 \mathrm{~b}^{*}$ & $0 \mathrm{a}^{*}$ & $24.7 b^{*}$ & $0 a^{*}$ & $0 \mathrm{a}$ & $0 \mathrm{a}$ & $0 \mathrm{a}$ & $0 \mathrm{a}$ \\
\hline Basamid (200) & 0 & 0 & 0 & 0 & $0 \mathrm{a}$ & $0 \mathrm{a}$ & $0 \mathrm{a}$ & $0 \mathrm{a}$ & $0 \mathrm{a}$ & $0 \mathrm{a}$ & $0 \mathrm{a}$ & $0 \mathrm{a}$ \\
\hline Basamid (160) & 0 & 0 & 0 & 0 & $0 \mathrm{a}$ & $0 \mathrm{a}$ & $0 \mathrm{a}$ & $0 \mathrm{a}$ & $0 a^{*}$ & $19.1 \mathrm{~b}^{*}$ & $0 \mathrm{a}$ & $0 \mathrm{a}$ \\
\hline Basamid (100) & 0 & 0 & 0 & 0 & $13 b^{\text {ns }}$ & $17.9 \mathrm{~b}^{\mathrm{ns}}$ & $36.4 \mathrm{c}^{*}$ & $9.3 \mathrm{~b}^{*}$ & $1.2 \mathrm{a}^{*}$ & $20.4 \mathrm{~b}^{*}$ & $18.5 \mathrm{~b}^{\mathrm{ns}}$ & $16.7 \mathrm{~b}^{\mathrm{ns}}$ \\
\hline Control & 100 & 100 & 100 & 100 & $100 \mathrm{c}$ & $100 \mathrm{c}$ & $100 \mathrm{~d}$ & $100 \mathrm{c}$ & $100 \mathrm{~b}$ & $100 \mathrm{c}$ & $100 \mathrm{c}$ & $100 \mathrm{c}$ \\
\hline
\end{tabular}

${ }^{y}$ Each value represents the mean of three replicates, each consisting of 54 infected carnation pieces. Recovery values followed by different letters within each column or by an asterisk $(*)$ within each row are significantly different according to Fisher's least significant difference test $(\alpha=0.05)$; ns $=$ not significant.

z Rates: liters/hectare for Divapan or kilograms/hectare for Basamid.

Table 8. Disease incidence (\%) of crown and root rot caused by Calonectria pauciramosa and $C$. polizzii on red clover seedlings grown in peat substrate mixed with carnation leaf pieces colonized by pathogen microsclerotia retrieved from both fumigated and untreated plots ${ }^{\mathrm{y}}$

\begin{tabular}{|c|c|c|c|c|c|c|c|c|c|c|c|c|}
\hline \multirow[b]{3}{*}{ Treatment, rate $\mathrm{z}^{\mathrm{z}}$} & \multicolumn{6}{|c|}{ Infectivity of $C$. polizzii } & \multicolumn{6}{|c|}{ Infectivity of $C$. pauciramosa } \\
\hline & \multicolumn{2}{|c|}{$\begin{array}{c}\text { Experiment } \\
\text { III }\end{array}$} & \multicolumn{2}{|c|}{ Experiment IV } & \multicolumn{2}{|c|}{ Experiment V } & \multicolumn{2}{|c|}{$\begin{array}{c}\text { Experiment } \\
\text { III }\end{array}$} & \multicolumn{2}{|c|}{ Experiment IV } & \multicolumn{2}{|c|}{ Experiment V } \\
\hline & VIF & TIF & VIF & TIF & VIF & TIF & VIF & TIF & VIF & TIF & VIF & TIF \\
\hline Divapan (400) & 0.0 & 0.0 & $0.0 \mathrm{a}$ & $0.0 \mathrm{a}$ & $0.0 \mathrm{a}$ & $0.0 \mathrm{a}$ & 0.0 & 0.0 & $0.0 \mathrm{a}$ & $0.0 \mathrm{a}$ & $0.0 \mathrm{a}$ & $0.0 \mathrm{a}$ \\
\hline Divapan (350) & 0.0 & 0.0 & $0.0 \mathrm{a}$ & $0.0 \mathrm{a}$ & $0.0 \mathrm{a}$ & $0.0 \mathrm{a}$ & 0.0 & 0.0 & $0.0 \mathrm{a}$ & $0.0 \mathrm{a}$ & $0.0 \mathrm{a}$ & $0.0 \mathrm{a}$ \\
\hline Divapan (250) & 0.0 & 0.0 & $15.8 \mathrm{~b}^{*}$ & $0.0 \mathrm{a}^{*}$ & $0.0 \mathrm{a}$ & $0.0 \mathrm{a}$ & 0.0 & 0.0 & $28.6 b^{*}$ & $0.0 \mathrm{a}^{*}$ & $0.0 \mathrm{a}$ & $0.0 \mathrm{a}$ \\
\hline Basamid (200) & 0.0 & 0.0 & $0.0 \mathrm{a}$ & $0.0 \mathrm{a}$ & $0.0 \mathrm{a}$ & $0.0 \mathrm{a}$ & 0.0 & 0.0 & $0.0 \mathrm{a}$ & $0.0 \mathrm{a}$ & $0.0 \mathrm{a}$ & $0.0 \mathrm{a}$ \\
\hline Basamid (160) & 0.0 & 0.0 & $0.0 \mathrm{a}$ & $0.0 \mathrm{a}$ & $0.0 \mathrm{a}^{*}$ & $28.1 \mathrm{~b}^{*}$ & 0.0 & 0.0 & $0.0 \mathrm{a}$ & $0.0 \mathrm{a}$ & $0.0 \mathrm{a}$ & $0.0 \mathrm{a}$ \\
\hline Basamid (100) & 0.0 & 0.0 & $17.2 \mathrm{~b}$ & $20.5 \mathrm{~b}$ & $4.2 \mathrm{a}^{*}$ & $27.6 b^{*}$ & 0.0 & 0.0 & $40.5 c^{*}$ & $13.6 b^{*}$ & $21.2 \mathrm{~b}$ & $23.4 \mathrm{~b}$ \\
\hline Control & 88.5 & 89.6 & $90.3 \mathrm{c}$ & $85.7 \mathrm{c}$ & $93.8 \mathrm{~b}$ & $89.2 \mathrm{c}$ & 90.3 & 87.4 & $88.4 \mathrm{~d}$ & $84.6 \mathrm{c}$ & $92.8 \mathrm{c}$ & $87.2 \mathrm{c}$ \\
\hline
\end{tabular}

${ }^{\mathrm{y}}$ Data presented are means of three replications (each consisting of 42 to 55 young red clover seedlings). Arcsine ( $\sin ^{-1}$ square root $x$ ) transformation was used on percentage data prior to analysis; untransformed data are presented. VIF = virtually impermeable film and TIF = totally impermeable film. Disease incidence values followed by different letters within each column or by * within each row are significantly different according to Fisher's least significant difference test $(\alpha=0.05)$. Infectivity levels of fumigated pathogen microsclerotia are always compared with those of relative controls.

${ }^{\mathrm{z}}$ Rates: liters/hectare for Divapan or kilograms/hectare for Basamid. 
and C. pauciramosa independent of fumigant, rate, and film. In experiment IV, all of the treatments were effective in eliminating the inocula of C. polizzii and C. pauciramosa, except the lowest rates of Basamid (DZ) (under both films) and Divapan (MS) (under VIF), which significantly reduced the viable inocula of each species compared with control. At these rates, TIF increased the efficacy of fumigant treatments compared with VIF. In the last experiment (V), only Basamid (DZ) failed to kill all of the viable microsclerotia of these two Calonectria spp. when applied at $100 \mathrm{~kg} / \mathrm{ha}$ and of $C$. polizzii at $160 \mathrm{~kg} / \mathrm{ha}$ under TIF. Significant differences related to fumigant rate and film were observed for Calonectria spp. survival but TIF did not improve the efficacies of treatments when compared with VIF.

Evaluation of the viability of $C$. polizzii and $C$. pauciramosa microsclerotia on red clover following fumigation treatments. Microsclerotia infectivity rates on young red clover seedlings in experiments III, IV, and V are reported in Table 8. In experiment III, no Calonectria infections were observed on preinfected carnation debris after retrieval from the fumigated plots. This corroborated the pathogen survival rate of zero. The disease incidence was strongly correlated to the survival of Calonectria microsclerotia. Thus, in experiment IV, Calonectria infections were only found on infected debris recovered from plots treated with Basamid (DZ) under VIF or TIF at a rate of $100 \mathrm{~kg} / \mathrm{ha}$ and Divapan (MS) under VIF at 250 liters/ha. Calonectria infections on red clover were only obtained from microsclerotia exposed to fumigation at a rate of $160 \mathrm{~kg} / \mathrm{ha}$ under TIF and at $100 \mathrm{~kg} / \mathrm{ha}$ under both films. The pairwise combinations of the significant differences between treatments were similar to those observed for the survival experiments.

\section{Discussion}

This article provides initial information on the efficacies of DZ and MS fumigants applied at reduced rates under low-permeability films under nursery conditions against 19 Calonectria spp., representative of a worldwide population.

In the greenhouse experiments, both DZ and MS were effective in reducing exotic Calonectria microsclerotia viability, although some differences in their performances were observed between experiments. Both Basamid (DZ) and Divapan (MS), when applied at $160 \mathrm{~kg} /$ ha and 400 liters/ha concentration and greater, respectively, under either film were effective in eradicating Calonectria microsclerotia in soil, except for C. hongkongensis (viable with Divapan at up to 700 liters/ha and Basamid up to $200 \mathrm{~kg} / \mathrm{ha}$ ), C. sulawesiensis (viable with Divapan at up to 400 liters/ha), and $C$. variabilis LPF220 and C. naviculata (viable with Basamid at up to 160 and $200 \mathrm{~kg} / \mathrm{ha}$, respectively). At lower rates, there was greater variability in microsclerotia variability among most Calonectria isolates in all of the experiments. In general, TIF enhanced fumigant performance. Because high inter- and intraspecific variability of responses to fumigants was detected among Calonectria spp. or isolates, the correct identification of target Calonectria spp. could play an important role in the choice of fumigant and application rate.

The field data confirmed the efficacy of the highest rates of both fumigants against $C$. polizzii and $C$. pauciramosa, as similarly reported by Polizzi et al. (2014). In comparison with a previous study that utilized higher labeled rates (Divapan and Basamid at 400, 700, and 1,000 liters/ha and 200,400 , and $500 \mathrm{~kg} / \mathrm{ha}$, respectively), the reduced rates (defined as less than the labeled rate) applied under VIF and TIF were also effective in reducing both endemic Calonectria spp. in soil. In addition, the efficacy of the fumigant treatment appeared to be strongly influenced by the season and, to a lesser extent, by the film used. Indeed, no viable inocula were retrieved from any of the fumigated plots in the July experiment. In the remaining experiments (in November and May), the lowest rates of fumigants were less effective and did not eliminate viable inocula of either pathogen. Moreover, TIF did not always improve the efficacy of the treatment when compared with VIF. The highest Calonectria infection rates were obtained from inocula retrieved from untreated plots and, to a lesser extent, from plots treated at the lowest fumigant rates.

The current labeled rates are 300 liters/ha in open fields and 700 to 1,200 liters/ha in greenhouses for Divapan 51 while the rates for Basamid range from 300 to $500 \mathrm{~kg} / \mathrm{ha}$ (at a 15 -to 20 -cm depth) for some pathogens to 500 to $700 \mathrm{~kg} / \mathrm{ha}$ (at a 25 - to 30 -cm depth) for other pathogens. Our data clearly showed the possibility of reducing the application rates by up to $160 \mathrm{~kg} / \mathrm{ha}$ for Basamid (DZ) and 400 liters/ha for Divapan (MS) against all Calonectria inocula in soil or substrate used for ornamental and forestry crops when using low-permeability films (VIF or TIF). High rates of MS should be applied only for C. hongkongensis, $C$. sulawesiensis, $C$. variabilis, and $C$. naviculata. The use of Basamid (DZ) applied at $100 \mathrm{~kg} / \mathrm{ha}$ and Divapan (MS) at 250 liters/ha in July could be encouraged for the eradication of endemic $C$. polizzii and $C$. pauciramosa under nursery conditions in the Mediterranean Basin. DZ and MS have been previously reported as effective against other soilborne pathogen inocula but at higher rates than those reported in this article (Wang et al. 2006; Weiland et al. 2011, 2013).

TIF is less permeable than VIF, and it allows a decrease in fumigant application rates by 40 to $50 \%$ without sacrificing fumigant efficacy (Fennimore and Ajwa 2011; Gao et al. 2013; McAvoy and Freeman 2013). However, the fumigant efficacy in our study was not always significantly greater under TIF compared with VIF. Nevertheless, TIF could be particularly attractive because it decrease gas emissions. High-permeability films (low-density and high-density polyethylene) should be replaced by low-permeability films to reduce or kill Calonectria inocula as well as already was reported for other soilborne fungal pathogens (Cabrera et al. 2015; Chamorro et al. 2016) because they are more environmentally friendly (Gao et al. 2011) and allow for the use of lower fumigant rates.

This investigation indicated that fumigation at reduced rates under low-permeability films is an appropriate option to reduce or kill soilborne Calonectria spp. before growing seedlings, propagating plants, or replanting pot-grown plants in nurseries. Because the most widely used potting substrate is derived from mixture of local (volcanic) soil with commercial peat and mineral constituents, the sustainable disinfection of soil or substrate should be encouraged on a large scale as an additional agronomic practice in nursery production.

\section{Literature Cited}

Aiello, D., Cirvilleri, G., Polizzi, G., and Vitale, A. 2013. Effects of fungicide treatments for the control of epidemic and exotic Calonectria diseases in Italy. Plant Dis. 97:37-43

Alfenas, R. F., Pereira, O. L., Jorge, V. L., Crous, P. W., and Alfenas, A. C. 2013. A new species of Calonectria causing leaf blight and cutting rot of three forest tree species in Brazil. Trop. Plant Pathol. 38:513-521.

Cabrera, J. A., Hanson, B. D., Gerik, J. S., Gao, S., Qin, R., and Wang, D. 2015. Pre-plant soil fumigation with reduced rates under low permeability films for nursery production, orchard and vineyard replanting. Crop Prot. 75:34-39.

Chamorro, M., Seijo, T. E., Noling, J. C., De los Santos, B., and Peres, N. A. 2016. Efficacy of fumigant treatments and inoculum placement on control of Macrophomina phaseolina in strawberry beds. Crop Prot. 90:163-169.

Chong, C. 2005. Experiences with wastes and composts in nursery substrates. HortTechnology 15:739-747.

Cinquerrui, A., Polizzi, G., Aiello, D., and Vitale, A. 2017. Integrated management for the reduction of Calonectria infections in ornamental nurseries. Plant Dis. 101:165-169.

Crous, P. W. 2002. Taxonomy and Pathology of Cylindrocladium (Calonectria) and Allied Genera. American Phytopathological Society, St. Paul, MN.

Crous, P. W., Groenewald, J. Z., Riséde, J. M., Simoneau, P., and Hide, K. D. 2006. Calonectria species and their Cylindrocladium anamorphs: Species with clavate vesicles. Stud. Mycol. 55:213-226.

Crous, P. W., Groenewald, J. Z., Risède, J. M., Simoneau, P., and Hywel-Jones, N. 2004. Calonectria species and their Cylindrocladium anamorphs: species with sphaeropedunculate vesicles. Stud. Mycol. 50:415-430.

Crous, P. W., Janse, B. J. H., Victor, D., Marais, G. F., and Alfenas, A. C. 1993a. Characterization of some Cylindrocladium species with three-septate conidia using morphology, isozyme banding patterns and DNA polymorphisms. Syst. Appl. Microbiol. 16:266-273.

Crous, P. W., Phillips, A. J. L., and Wingfield, M. J. 1991. The genera Cylindrocladium and Cylindrocladiella in South Africa, with special reference to forestry nurseries. S. Afr. For. J. 157:69-85.

Crous, P. W., Wingfield, M. J., and Alfenas, A. 1993b. Additions to Calonectria. Mycotaxon 46:217-234

Crous, P. W., Wingfield, M. J., Alfenas, A. C., and Silveira, S. F. 1994 Cylindrocladium naviculatum sp. nov., and two new vesciculate hyphomycete genera, Falcocladium and Vesiculomyces. Mycotaxon 50:441-458.

Daughtrey, M. L., and Benson, D. M. 2005. Principles of plant health management for ornamental plants. Annu. Rev. Phytopathol. 43:141-169.

European Commission. 2012. Commission Implementing Regulation (EU) No $359 / 2012$ of 25 April 2012. Online publication. Official Journal of the 
European Union. http://eur-lex.europa.eu/legal-content/IT/TXT/?uri=CELEX: 32012R0359

European Parliament and the Council of the European Union. 2009. Directive 2009/128/EC of the European Parliament and of the Council of 21 October 2009. Online publication. Official Journal of the European Union. http://eur-lex. europa.eu/LexUriServ/LexUriServ.do?uri=OJ:L:2009:309:0071:0086:en:PDF

Fennimore, S. A., and Ajwa, H. A. 2011. Totally impermeable film retains fumigants, allowing lower application rates in strawberry. Calif. Agric. 65:211-215.

Fisher, N. L., Burgess, L. W., Toussoun, T. A., and Nelson, P. E. 1982. Carnation leaves as a substrate and for preserving cultures of Fusarium species. Phytopathology 72:151-153.

Gao, S., Ajwa, H., Qin, R., Stanghellini, M., and Sullivan, D. 2013. Emission and transport of 1,3-dichloropropene and chloropicrin in a large field tarped with VaporSafe $^{\text {TM }}$ TIF. Environ. Sci. Technol. 47:405-411.

Gao, S., Hanson, B. D., Wang, D., Browne, G. T., Qin, R., Ajwa, H., and Yates, S. R. 2011. Methods evaluated to minimize emissions from preplant soil fumigation. Calif. Agric. 65:41-46.

Guarnaccia, V., Aiello, D., Polizzi, G., Perrone, G., Stea, G., and Vitale, A. 2014. Emergence of prochloraz resistant populations of Calonectria pauciramosa and Calonectria polizzii in ornamental nurseries of Southern Italy. Plant Dis. 98:344-350.

Harman, G. E. 2000. Myths and dogmas of biocontrol: Changes in perceptions derived from research on Trichoderma harzianum T-22. Plant Dis. 84:377-393.

Henricot, B., and Culham, A. 2002. Cylindrocladium buxicola, a new species affecting Buxus spp., and its phylogenetic status. Mycologia 94:980-997.

LaMondia, J. A. 2014. Fungicide efficacy against Calonectria pseudonaviculata, causal agent of boxwood blight. Plant Dis. 98:99-102.

LaMondia, J. A. 2015. Management of Calonectria pseudonaviculata in boxwood with fungicides and less susceptible host species and varieties. Plant Dis. 99:363-369.

Lombard, L., Crous, P. W., Wingfield, B. D., and Wingfield, M. J. 2010a. Species concepts in Calonectria (Cylindrocladium). Stud. Mycol. 66:1-13.

Lombard, L., Crous, P. W., Wingfield, B. D., and Wingfield, M. J. 2010b. Multigene phylogeny and mating tests reveal three cryptic species related to Calonectria pauciramosa. Stud. Mycol. 66:15-30.

Lombard, L., Crous, P. W., Wingfield, B. D., and Wingfield, M. J. 2010c. Phylogeny and systematics of the genus Calonectria. Stud. Mycol. 66:31-69.

Lombard, L., Polizzi, G., Guarnaccia, V., Vitale, A., and Crous, P. W. 2011. Calonectria spp. causing leaf spot, crown and root rot of ornamental plants in Tunisia. Persoonia 27:73-79.

Lombard, L., Rodas, C. A., Crous, P. W., Wingfield, B. D., and Wingfield, M. J. 2009. Calonectria (Cylindrocladium) species associated with dying Pinus cuttings. Persoonia 23:41-47.

McAvoy, T. P., and Freeman, J. H. 2013. Retention of the soil fumigant dimethyl disulfide by virtually and totally impermeable film mulches. HortScience 48:1154-1158.

Noble, R., and Roberts, S. J. 2004. Eradication of plant pathogens and nematodes during composting: A review. Plant Pathol. 53:548-568

Phipps, P. M., and Beute, M. K. 1979. Population dynamics of Cylindrocladium crotalariae microsclerotia in naturally-infested soil. Phytopathology 69:240-243.

Polizzi, G., Aiello, D., Guarnaccia, V., Cinquerrui, A., Formica, P. T., Vitale, A., and Myrta, A. 2014. Effects of label and sub-label rates of dazomet and methamsodium on survival of Calonectria microsclerotia. Acta Hortic. 1044:389-394.

Polizzi, G., and Crous, P. W. 1999. Root and collar rot of milkwort caused by Cylindrocladium pauciramosum, a new record for Europe. Eur. J. Plant Pathol. 105:407-411.

Polizzi, G., Grasso, F. M., Vitale, A., and Aiello, D. 2007a. First occurrence of Calonectria leaf spot on Mexican blue palm in Italy. Plant Dis. 91:1052.

Polizzi, G., Vitale, A., Aiello, D., Castello, I., Guarnaccia, I., and Parlavecchio, G. 2009. First record of crown and root rot caused by Cylindrocladium pauciramosum on brush cherry in Italy. Plant Dis. 93:547.
Polizzi, G., Vitale, A., Aiello, D., Dimartino, M. A., and Parlavecchio, G. 2007b. First report of damping-off and leaf spot caused by Cylindrocladium scoparium on different accessions of bottlebrush cuttings in Italy. Plant Dis. 91:769.

Polizzi, G., Vitale, A., Aiello, D., Guarnaccia, V., Crous, P. W., and Lombard, L. 2012. First report of Calonectria ilicicola causing a new disease on Laurus (Laurus nobilis) in Europe. J. Phytopathol. 160:41-44.

Polizzi, G., Vitale, A., Aiello, D., and Parlavecchio, G. 2006a. First record of crown and root rot caused by Cylindrocladium pauciramosum on California lilac in Italy. Plant Dis. 90:1459.

Polizzi, G., Vitale, A., Castello, I., Groenewald, J. Z., and Crous, P. W. 2006b. Cylindrocladium leaf spot, blight, and crown rot, new diseases of mastic tree seedlings caused by Cylindrocladium scoparium. Plant Dis. 90:1110.

Schoch, C. L., Crous, P. W., Wingfield, B. D., and Wingfield, M. J. 1999. The Cylindrocladium candelabrum species complex includes four distinct mating populations. Mycologia 91:286-298.

Thies, W. F., and Patton, R. F. 1970. The biology of Cylindrocladium scoparium in Wisconsin in forest tree nurseries. Phytopathology 60:1662-1668.

Victor, D., Crous, P. W., Janse, B. J. H., and Wingfield, M. J. 1997. Genetic variation in Cylindrocladium floridanum and other morphologically similar Cylindrocladium species. Syst. Appl. Microbiol. 20:268-285.

Vitale, A., Aiello, D., Castello, I., Dimartino, M. A., Parlavecchio, G., and Polizzi, G. 2009a. Severe outbreak of crown rot and root rot caused by Cylindrocladium pauciramosum on strawberry tree in Italy. Plant Dis. 93:842.

Vitale, A., Aiello, D., Castello, I., Parlavecchio, G., and Polizzi, G. 2008. First report of crown rot and root rot caused by Cylindrocladium pauciramosum on Feijoa (Feijoa sellowiana) in Italy. Plant Dis. 92:1590.

Vitale, A., Aiello, D., Castello, I., and Polizzi, G. 2009b. First report of benzimidazole-resistant isolates of Cylindrocladium scoparium in Europe. Plant Dis. 93:110.

Vitale, A., Castello, I., D'Emilio, A., Mazzarella, R., Perrone, G., Epifani, F., and Polizzi, G. 2013a. Short-term effects of soil solarization in suppressing Calonectria microsclerotia. Plant Soil 368:603-617.

Vitale, A., Cirvilleri, G., Castello, I., Aiello, D., and Polizzi, G. 2012. Evaluation of Trichoderma harzianum strain T22 as biological control agent of Calonectria pauciramosa. BioControl 57:687-696.

Vitale, A., Crous, P. W., Lombard, L., and Polizzi, G. 2013b. Calonectria diseases on ornamental plants in Europe and the Mediterranean basin: An overview. J. Plant Pathol. 95:463-476.

Vitale, A., and Polizzi, G. 2008. First record of leaf spots and stem lesions on Pistacia lentiscus caused by Cylindrocladium pauciramosum and C. scoparium in Italy. Plant Pathol. 57:384.

Waipara, N. W., Di Menna, M. E., Cole, A. L. J., and Skipp, R. A. 1996 Pathogenicity of Cylindrocladium scoparium to pasture clover and grass species. Australas. Plant Pathol. 25:205-211.

Walters, D. 2009. Disease Control Crops: Biological and Environmentally Friendly Approaches. D. Walters, ed. Wiley-Blackwell, Oxford, UK.

Wang, D., Fraedrich, S. W., Juzwik, J., Spokas, K., Zhang, Y., and Koskinen, W. C. 2006. Fumigant distribution in forest nursery soils under water seal and plastic film after application of dazomet, metam-sodium and chloropicrin. Pest Manage. Sci. 62:263-273.

Weiland, J. E., Leon, A. L., Edmonds, R. L., Littke, W. R., Browning, J. E., Davis, A., Beck, B. R., Miller, T. W., Cherry, M. L., and Rose, R. 2011. The effects of methyl bromide alternatives on soil and seedling pathogen populations, weeds, and seedling morphology in Oregon and Washington forest nurseries. Can. J. For. Res. 41:1885-1896.

Weiland, J. E., Littke, W. R., and Haase, D. L. 2013. Forest nurseries face critical choices with the loss of methyl bromide fumigation. Calif. Agric. 67:153-161. 